\title{
Courant-Friedrichs' Hypothesis and Stability of the Weak Shock Wave Satisfying the Lopatinski Condition
}

\author{
Dmitry Tkachev, Alexander Blokhin \\ Mechanics and Mathematics Department, Novosibirsk State University, Sobolev Institute of Mathematics, Novosibirsk, Russia \\ Email: tkachev@math.nsc.ru, blokhin@math.nsc.ru
}

Received 2013

\begin{abstract}
We are studying the problem of a stationary supersonic flow of an inviscid non-heat-conducting gas in thermodynamical equilibrium onto a planar infinite wedge. It is known that theoretically this problem has two solutions: the solution with a strong shock wave (when the velocity behind the front of the shock wave is subsonic) and the solution with a weak shock wave (when, generally speaking, the velocity behind the front of the shock wave is supersonic). In the present paper, the case of a weak shock wave is studied. It is proved that if the Lopatinski condition for the shock wave is satisfied (in a weak sense), then the corresponding linearized initial boundary-value problem is well-posed, and its classical solution is found. In this case, unlike the case when the uniform Lopatinski condition holds, additional plane waves appear. It is shown that for compactly supported initial data the solution of the linearized problem converges in finite time to the zero solution. Therefore, for the case of a weak shock wave and when the Lopatinski condition holds in a weak sense these results complete the verification of the well-known Courant-Friedrichs' conjecture that the strong shock wave solution is unstable whereas the weak shock wave solution is stable.
\end{abstract}

Keywords: Weak Shock Wave; Asymptotic Stability (in the Sense of Lyapunov)

\section{Introduction}

It is well-known that the classical problem of a stationary supersonic flow of an inviscid non-heat-conducting gas onto an infinite plane wedge (with a sufficiently small angle $\sigma$ at the vertex) has two solutions: one of them corresponds to the case of a strong shock wave, when the components of the velocity vector $U_{0}: u_{0}, v_{0}$ behind the shock satisfies the inequality $u_{0}^{2}+v_{0}^{2}<c_{0}^{2} \quad\left(c_{0}\right.$ is the sound speed of the gas behind the shock wave), and the second corresponds to the case of a weak shock wave, that is $u_{0}^{2}+v_{0}^{2}>c_{0}^{2}[1-3]$ (see Figure 1).

In Figure 1 the angular coordinates $\theta_{s}, \theta_{w}$ determine strong and weak shocks respectively. The vector of flow velocity $U_{w}$ is parallel to the $U x$ axis.

Paradoxically, but in practice, in physical or numerical experiments, only the weak shock wave solution is actually realized. One possible explanation was suggested by Courant and Friedrichs [1]. They conjectured that the solution corresponding to a strong shock wave is unstable by Lyapunov whereas the weak shock wave solution is stable.

The Courant-Friedrichs' hypothesis was verified in [4-7] (but this conclusion was based only on some qualitative reasons). A strict mathematical justification (and this is very important) of this statement for the linearized problem appeared in recent years in [8-12]. Briefly speaking, it was shown in [8-12] that in the case of a strong shock wave (for compactly supported initial data!) the perturbation arrives to the wedge's vertex as time increases having the growth $r^{\alpha} \quad(\alpha<0)$ or a logarithmic growth in space variables, and this causes instability of the steady-state solution under consideration.

The situation with the weak shock wave is totally different. In this case the perturbation decays with time. Moreover, this solution is asymptotically stable by Lyapunov. It is assumed that in the both cases the shock satisfies the well-known uniform Lopatinski condition [13].

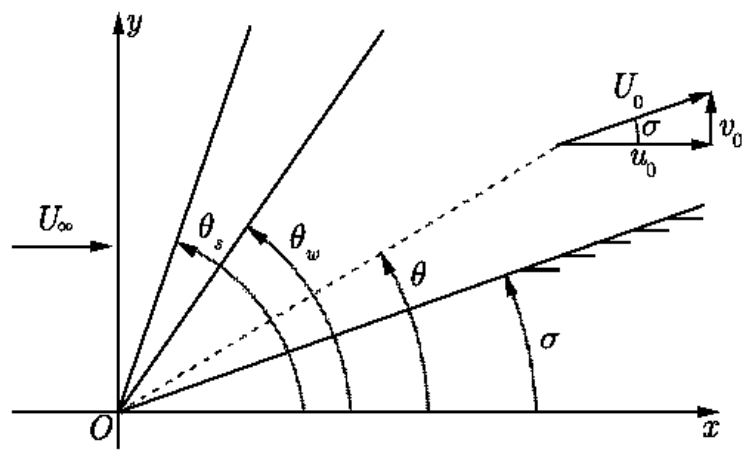

Figure 1. 
The present work is a continuation of [10]. We consider the more general case when the Lopatinski condition on the shock wave is satisfied only in a weak sense [13], i.e. the uniform Lopatinski condition can be violated. In particular, this makes finding the classical solution of this problem more difficult. The key point of the work is the analysis of an explicit form of the solution and we extensively use the technique developed in [10].

\section{Statement of the Original and the Auxiliary Problems and Formulation of Main Results}

The linear problem of finding a supersonic stationary gas flow onto a planar infinite wedge can be stated as follows [14]. We seek for a solution of the system of acoustic equations

$$
A U_{t}+B U_{x}+C_{\sigma} U_{y}=0
$$

in the domain $t, x>0, y>x \tan \sigma$ that satisfies the following boundary conditions at the shock wave $(x=0)$ and on the wedge $(y=x \tan \sigma)$ :

$$
\begin{gathered}
u_{1}+d u_{3}=0, u_{3}+u_{4}=0, u_{2}=\frac{\lambda}{\mu} F_{y}, \\
F_{t}+F_{y} \tan \sigma=\mu u_{3} ; \\
u_{2}=u_{1} \tan \sigma,
\end{gathered}
$$

and at $t=0$ it also satisfies the initial data

$$
U(0, x, y)=U_{0}(x, y), F(0, y)=F_{0}(y) .
$$

Here $U(t, x, y)=\left(u_{1}, u_{2}, u_{3}, u_{4}\right)^{\top}: u_{1}, u_{2}, u_{3}, u_{4}$ are smooth perturbations of the components of the velocity, the pressure and the entropy respectively; $x=F(t, y)$ is a small displacement of the discontinuity front, and

$$
F(t, 0)=F_{0}(0)=0 \text {, }
$$

that means that we consider the case of a shock wave attached to the wedge's vertex. It is also assumed that the components of the vector $U_{0}(x, y)$ of initial data are compactly supported, i.e.

$$
\operatorname{suppu}_{0 i} \subset R_{+}^{2}=\{(x, y) \mid x, y>0\}, i=1,2,3,4 .
$$

The matrices $A, B, C_{,} \mathcal{C}_{a}$ read as follows:

$$
\begin{aligned}
& A=\operatorname{diag}\left(M^{2}, M^{2}, 1,1\right), B=\left(\begin{array}{cccc}
M^{2} & 0 & 1 & 0 \\
0 & M^{2} & 0 & 0 \\
1 & 0 & 1 & 0 \\
0 & 0 & 0 & 1
\end{array}\right), \\
& C=\left(\begin{array}{llll}
0 & 0 & 0 & 0 \\
0 & 0 & 1 & 0 \\
0 & 1 & 0 & 0 \\
0 & 0 & 0 & 0
\end{array}\right), C_{\sigma}=C+\tan \sigma A ;
\end{aligned}
$$

$M=\frac{u_{0}}{c_{0}}, M<1$ is the Mach number behind of the shock wave $\left(u_{0}, v_{0}=u_{0} \tan \sigma\right.$ are the components of the velocity vector of the stationary solution, $c_{0}$ is the downstream sound speed), and the physical constants $d, \lambda, \mu$ were described in detail in [14]. These constants depend on the components of the piecewise constant solution corresponding to the step shock as well as on the state equation of gas $p=p(\rho, s) \quad$ ( $\rho$ is the density of the gas and $s$ is the entropy) and the equation of the Hugoniot adiabat.

If the solution of problem (1)-(4) is continuous up to the boundary $x=0, y=x \tan \sigma$, then, in view of (5), it follows from the boundary conditions (2), (3) that the following compatibility condition should be fulfilled at the edge $t \geq 0, x=y=0$ :

$$
\left(\lambda+d \tan ^{2} \sigma\right) u_{3}(t, 0,0)=0, t \geq 0,
$$

i.e. if $D_{1}=\lambda+d \tan ^{2} \sigma \neq 0$, then

$$
U(t, 0,0)=0, t \geq 0 \text {. }
$$

\section{Remark 1.}

We have formulated the initial boundary-value problems (1)-(5) for the case when the gas flow in a neighborhood of the wedge with shock wave directed along the $O y$ - axis is fixed as the main solution (see Figure 2).

In this paper we consider the case of a weak shock wave, i.e. the case when

$$
M_{0}=\sqrt{\frac{u_{0}^{2}+v_{0}^{2}}{c_{0}^{2}}}=\frac{M}{\cos \sigma}>1 .
$$

Further we will also assume that the state equation of gas is such that

$$
\lambda<0, d>-\frac{1}{M}, d\left(1-M^{2}\right)+\lambda M^{2}<0,
$$

The coefficient $D_{1} \neq 0$ and, moreover, $D_{1}>0$ (for example, this is true for the polytropic gas [10]; some examples of state equations satisfying (8) are considered in [15]).

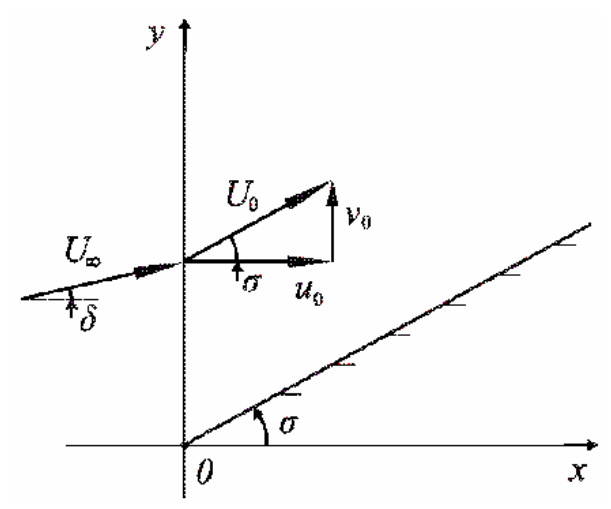

Figure 2. 
Assume that the solution of problems (1)-(5) is not just continuous but also has second derivatives which are continuous up to the boundary of the domain. Taking mixed derivatives we can reduce problem (1)-(5) with condition (6) to the following initial boundary-value problem for the component $u_{3}$ (the pressure). In the domain $t>0, x>0, y>x \tan \sigma$ we seek for a smooth solution of the wave equation

$$
\left\{M^{2} L_{1}^{2}-L_{2}^{2}-\left(\frac{\partial}{\partial y}\right)^{2}\right\} u_{3}=0,
$$

that satisfies the following boundary conditions at the shock wave $(x=0)$ and on the wedge $(y=x \tan \sigma)$ :

$$
\begin{gathered}
\left\{m L_{1}^{2}+n L_{2}^{2}-\frac{\beta}{M^{2}} L_{1} L_{2}\right\} u_{3}=0 ; \\
\left\{\cos \sigma\left(\frac{\partial}{\partial y}\right)-\sin \sigma\left(\frac{\partial}{\partial x}\right)\right\} u_{3}=0 ; \\
u_{3}(t, 0,0)=0,
\end{gathered}
$$

and it also satisfies the initial data for $t=0$ :

$$
\left.u_{3}\right|_{t=0}=u_{0}(x, y),\left.\left(u_{3}\right)_{t}\right|_{t=0}=u_{1}(x, y)
$$

(note that the derivative $\left.\left(u_{3}\right)_{t}\right|_{t=0}$ can be found from the third equation of system (1)).

In equations (9), (10) we have used the following notations:

$$
\begin{aligned}
& L_{1}=\frac{1}{\beta} l_{1}, l_{1}=\frac{\partial}{\partial t}+\tan \sigma \frac{\partial}{\partial y}, L_{2}=\beta \frac{\partial}{\partial x}-\frac{M^{2}}{\beta} l_{1}, \\
& \beta^{2}=1-M^{2}, n=-\frac{\lambda}{\beta}, m=\beta d+\frac{\lambda M^{2}}{\beta} .
\end{aligned}
$$

The converse also holds, i.e. for each solution $u_{3}$ of problems (9)-(13) we can uniquely find a corresponding smooth solution $U(t, x, y), \quad F(t, y)$ of problem (1)-(6). This fact can be proved by the same way as for the case of a half-plane $x>0$ (see [14]).

Unlike [10], we assume that problem (9) - (13) satisfies the Lopatinski condition [13] at the boundary $x=0$ only in a weak sense, i.e. the uniform Lopatinski condition $m>0, n>0$ (see [14]) or equivalently the condition

$$
\lambda<0, d>-\frac{\lambda M^{2}}{\beta^{2}} .
$$

can be violated.

In Figure 3 we shade the domain where the Lopatinski condition is satisfied (in a weak sense).

This domain is described by the two systems of inequalities

$$
\left\{\begin{array} { c } 
{ d > - \frac { 1 } { M } , d < - \frac { \lambda M ^ { 2 } } { \beta ^ { 2 } } , } \\
{ \lambda < 0 }
\end{array} \text { and } \left\{\begin{array}{c}
d<-\frac{1}{M}, \\
d>-\frac{\lambda M^{2}}{\beta^{2}}
\end{array}\right.\right.
$$

The strait line $d=\frac{1}{M}+\frac{\rho_{\infty}}{\rho_{0}} \lambda$ corresponds to the gas dynamic case, and $\rho_{\infty}$ and $\rho_{0}$ are the densities ahead and behind of the shock front respectively.

Remark 2.

For the state equations from [15] the point $(\lambda, d)$ lies in the second quadrant and $d<-\frac{\lambda M^{2}}{\beta^{2}}$.

We point out that the uniform Lopatinski condition holds at the boundary $y=x \tan \sigma$.

Now we make a convenient change of coordinates by setting

$$
x^{\prime}=x, y^{\prime}=y-x \tan \sigma .
$$

We shall drop the primes in what follows.

Then problem (9)-(13) takes the following form:

$$
\begin{gathered}
\left\{M^{2}\left(\frac{\partial}{\partial t}+\frac{\partial}{\partial x}\right)^{2}-\left(\frac{\partial}{\partial x}-\tan \sigma \frac{\partial}{\partial y}\right)^{2}\right. \\
\left.-\left(\frac{\partial}{\partial y}\right)^{2}\right\} u=0, \quad t, x, y>0 ; \\
\left\{( \frac { \partial } { \partial t } + \operatorname { t a n } \sigma \frac { \partial } { \partial y } ) \left[\frac{\partial}{\partial t}+\frac{\partial}{\partial x}+d\left(\frac{\partial}{\partial t}+\tan \sigma \frac{\partial}{\partial y}\right)\right.\right. \\
\left.\left.-\frac{1}{M^{2}}\left(\frac{\partial}{\partial x}-\tan \sigma \frac{\partial}{\partial y}\right)\right]+\lambda\left(\frac{\partial}{\partial y}\right)^{2}\right\} u=0, \quad x=0 ; \\
\left(\frac{\partial}{\partial y}-\sin \sigma \cos \sigma \frac{\partial}{\partial x}\right) u=0, \quad y=0 ; \\
u(t, 0,0)=0 ; \\
\left.u\right|_{t=0}=u_{0}(x, y),\left.u_{t}\right|_{t=0}=u_{1}(x, y)
\end{gathered}
$$

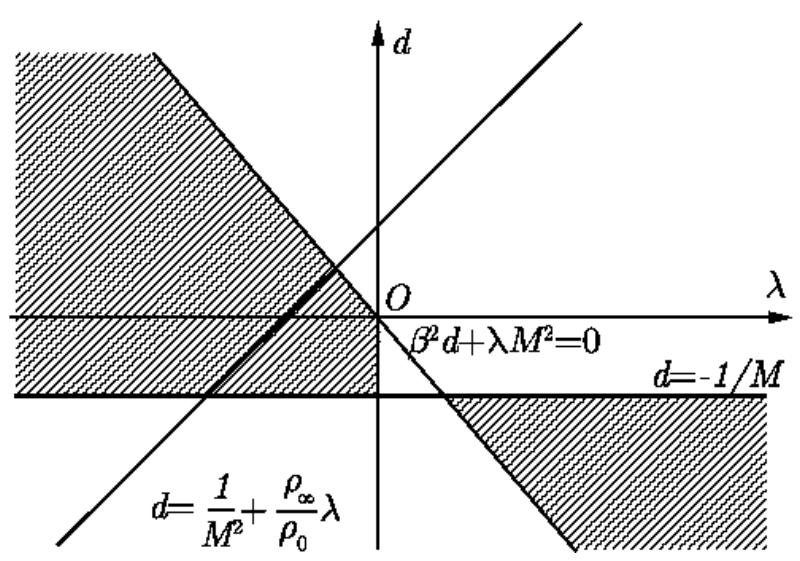

Figure 3. 
(we also drop the subscript 3 by the unknown function $\left.u_{3}\right)$.

Since problems (1)-(5) and (17)-(21) are equivalent, it is sufficient to state our main results for the solution $u(t, x, y)$ of problems (17)-(21).

In addition, we also assume that the following property characterizing the behavior of the solution as $t, x \rightarrow+\infty$ holds: there exist parameters $s_{0}$ and $p_{0}$ such that the function $e^{-s_{0} t} e^{-p_{0} x} u(t, x, y)$ is bounded as $t, x \rightarrow+\infty$ for each fixed $y>0$, i.e.

$$
\begin{aligned}
& u(t, x, y)=O\left(e^{s_{0} t+p_{0} x}\right), \\
& t, x \rightarrow+\infty, y>0 \text { is fixed. }
\end{aligned}
$$

Let us introduce the notations

$$
\begin{aligned}
\Delta=M_{0}^{2}-1, B_{0} & =\frac{\sqrt{\Delta}}{\tan \sigma}\left(B_{0}<1\right), D_{1}=\lambda+d \tan ^{2} \sigma, \\
D_{2} & =\frac{\sqrt{\Delta}}{M^{2}} \tan \sigma, L=\frac{1-B_{0}}{1+B_{0}}
\end{aligned}
$$

and assume that the problem's parameters are linked by the relation

$$
\frac{\left|D_{2}-D_{1}\right|}{D_{2}+D_{1}}<L
$$

The following results hold.

\section{Theorem 1.}

If the initial data for problem (17)-(21) are compactly supported (i.e. $\operatorname{suppu}_{0}, u_{1} \subset R_{+}^{2}$ ) and inequality (23) holds, then the classical solution of the problem exists, is unique and can be defined by formula

$$
\begin{aligned}
& \left.u(t, x, y)=\frac{8 \Delta}{\beta^{2} M^{2}}\left(\frac{\partial}{\partial y^{\prime \prime}}+B_{0} \frac{\partial}{\partial x^{\prime \prime}}\right) \int_{0}^{\left(x^{\prime \prime}+y^{\prime \prime}\right.}, \frac{B_{0}\left(x^{\prime \prime}+y^{\prime \prime}\right)}{1+B_{0}}\right) \\
& \div \bar{g}(t, \xi){ }_{t} E\left(t-\frac{M^{2}}{2 \Delta}\left(\sqrt{\Delta}\left(y^{\prime \prime}-B \xi\right)-\left(x^{\prime \prime}-\xi\right) \tan \sigma\right), /\right. \\
& \left.x^{\prime \prime}-\xi, y^{\prime \prime}-B \xi\right) d \xi-\frac{8 \Delta}{\beta^{2} M^{2}} \int_{0}^{\left(x^{\prime \prime}-y^{\prime \prime}, 0\right)} \bar{l}(t, \xi)_{t} / \\
& { }_{t} E\left(t-\frac{M^{2}}{2 \Delta}\left(\sqrt{\Delta} y^{\prime \prime}-\left(x^{\prime \prime}-\xi\right) \tan \sigma\right), x^{\prime \prime}-\xi, y^{\prime \prime}\right) d \xi \\
& -\frac{8 \Delta}{\beta^{2} M^{2}} \frac{\partial}{\partial y^{\prime \prime}} \int_{0}^{\left(x^{\prime \prime}-y^{\prime \prime}, 0\right)} \bar{f}(t, \xi)_{t} \\
& { }_{t} E\left(t-\frac{M^{2}}{2 \Delta}\left(\sqrt{\Delta} y^{\prime \prime}-\left(x^{\prime \prime}-\xi\right) \tan \sigma\right), /\right. \\
& \left.x^{\prime \prime}-\xi, y^{\prime \prime}\right) d \xi+8 \int_{\square O Q M_{0} P}\left(\tan \sigma u_{0 \xi}+\sqrt{\Delta} u_{0 \eta}\right) \\
& \times E\left(t-\frac{M^{2}}{2 \Delta}\left(\sqrt{\Delta}\left(y^{\prime \prime}-\eta\right)-\left(x^{\prime \prime}-\xi\right) \tan \sigma\right),\right. \\
& \left.x^{\prime \prime}-\xi, y^{\prime \prime}-\eta\right) d \xi d \eta+2 \frac{\partial}{\partial t} \int_{\square O Q M_{0} P} u_{0}(\xi, \eta) \\
& \times E\left(t-\frac{M^{2}}{2 \Delta}\left(\sqrt{\Delta}\left(y^{\prime \prime}-\eta\right)-\left(x^{\prime \prime}-\xi\right) \tan \sigma\right), /\right.
\end{aligned}
$$

$$
\begin{aligned}
& \left.x^{\prime \prime}-\xi, y^{\prime \prime}-\eta\right) d \xi d \eta+2 \int_{\square O Q M_{0} P} u_{1}(\xi, \eta) \\
& \times E\left(t-\frac{M^{2}}{2 \Delta}\left(\sqrt{\Delta}\left(y^{\prime \prime}-\eta\right)-\left(x^{\prime \prime}-\xi\right) \tan \sigma\right), /\right. \\
& \left.x^{\prime \prime}-\xi, y^{\prime \prime}-\eta\right) d \xi d \eta ; x^{\prime \prime}=2\left(y+x \frac{\tan \sigma}{\beta^{2}}\right), \quad y^{\prime \prime}=2 \frac{\sqrt{\Delta}}{\beta^{2}} x,
\end{aligned}
$$

the first integral is over the line $y^{\prime \prime}=B_{0} x^{\prime \prime}$, and the next two integrals are over the abscissa axis $y^{\prime \prime}=0$; in the last two integrals over the quadrangle $\square O Q M_{0} P$ we have the following coordinates: the point

$$
Q\left(x^{\prime \prime}-y^{\prime \prime}, 0\right), \text { the point } P\left(\frac{x^{\prime \prime}+y^{\prime \prime}}{2}, \frac{B_{0}\left(x^{\prime \prime}+y^{\prime \prime}\right)}{2}\right),
$$

and the point $M_{0}\left(x^{\prime \prime}, y^{\prime \prime}\right)$; the function $E(t, x, y)$ is the fundamental solution of the operator of equation (14); $u_{0}(x, y)$ and $u_{1}(x, y)$ are the initial data (where the coordinates $x, y$ are expressed through the variables $\left.x^{\prime \prime}, y^{\prime \prime}\right)$; the functions $\bar{g}\left(t, x^{\prime \prime}\right), \quad \bar{l}\left(t, x^{\prime \prime}\right), \quad \bar{f}\left(t, x^{\prime \prime}\right)$ are known, in particular, $\bar{f}\left(t, x^{\prime \prime}\right)$ is determined as follows:

$$
\bar{f}\left(t, x^{\prime \prime}\right)=\sum_{n=0}^{N\left(t, x^{\prime \prime}\right)} L_{p \rightarrow x^{\prime \prime}, s \rightarrow t}^{-1} H_{n}(p, s)
$$

where $N\left(t, x^{\prime \prime}\right)$ is a certain integer number, $N\left(t, x^{\prime \prime}\right) \geq 0$.

\section{Remark 3.}

We do not write down here formulae defining the functions $\bar{g}\left(t, x^{\prime \prime}\right), \bar{l}\left(t, x^{\prime \prime}\right), \bar{f}\left(t, x^{\prime \prime}\right)$ and $H_{n}(p, s)$ because of their awkwardness.

\section{Theorem 2.}

For problem (17)-(21) the trace

$$
\left.f\left(t, x^{\prime \prime}\right)\right|_{x^{\prime \prime}=2 y}=\left.u(t, x, y)\right|_{x=0}
$$

at the shock wave is a superposition of a finite number of cylindrical and plane waves. Namely, representation

$$
u(t, 0, y)=\left.\sum_{n=0}^{N(t, y)} L_{p \rightarrow x^{\prime \prime}, s \rightarrow t}^{-1} H_{n}(p, s)\right|_{x^{\prime \prime}=y} .
$$

takes place.

\section{Theorem 3.}

If $y \in K$ ( $K$ is a compact subset of the real half-axis), then there exists $t^{*}(K, \Omega) \quad(\Omega$ is the support of the initial data $u_{0}$ and $\left.u_{1}\right)$ such that $f(t, y) \equiv 0$ when $t \geq t^{*}(K, \Omega), \quad y \in K$.

\section{Conclusions}

So,

1) we prove on the linearized level that the solution with a weak shock is asymptotically stable (by Lyapunov) in the case when on the shock front the Lopatinski conditions is satisfied in a weak sense, i.e., the uniform Lopatinski condition is violated.

2) Moreover, for compactly supported initial data any 
solution of the linearized initial boundary value problem becomes stationary for a finite time.

Our result justifies the classical Courant-Friedrichs' hypothesis that the strong shock wave solution is unstable whereas the weak shock wave solution is stable (when the Lopatinski condition holds at least in a weak sense).

\section{Acknowledgments}

This research was supported by the Russian Foundation for Basic Research (grants No. 10-01-302-a and 11-0800286-a) and the Russian Ministry of Education (grant No. 14.B37.21.0355).

The authors are also grateful to A.V. Yegitov for the assistance in preparing the manuscript.

\section{REFERENCES}

[1] R. Courant and K. O. Friedrichs, "Supersonic Flow and Shock Waves," Intersc. Publ., New York, 1948.

[2] L. V. Ovsyannikov, "Lectures on Basic Gas Dynamics," Institute of Computer Studies, Moscow, Izhevsk, 2003.

[3] G. G. Chernyi, "Gas Dynamics,” Nauka, Moscow, 1988.

[4] A. I. Rylov, "On the Possible Modes of Flow Round Tapered Bodies of Finite Thickness at Arbitrary Supersonic Velocities of the Approach Stream," Journal of Applied Mathematic and Mechanics, Vol. 55, No. 1,1991, pp. 78-85.doi:10.1016/0021-8928(91)90065-3

[5] A. A. Nikolski, "Plane Vorticity Gas Flows," Teor. Issled. Mekhk. Zhidkosti i Gaza. Tr. Tsentral. Aero- i Gidrodinamich. Inst., Vol. 2122, 1984, pp. 74-85.

[6] B. L. Rozhdestvenskii, "Corrected Theory of Supersonic Flow of a Nonviscous Gas about a Wedge," Math Mod- eling Comput. Experiment 1, No. 1, 1993, pp. 15-18.

[7] B. M. Bulakh, "Nonlinear Conical Flow," Delft Univ. Press, Delft, 1985.

[8] A. M. Blokhin, D. L. Tkachev and L. O. Baldan, "Study of the Stability in the Problem on Flowing Around a Wedge. The Case of Strong Wave," Journal of Mathematical Analysis and Applications, Vol. 319, No. 1, 2006, pp. 248-277. doi:10.1016/j.jmaa.2005.10.023

[9] A. M. Blokhin, D. L. Tkachev and Y. Y. Pashinin, "Stability Condition for Strong Shock Waves in the Problem of Flow around an Infinite Plane Wedge," Nonlinear Analysis: Hybrid Systems, Vol. 2, 2008, pp. 1-17. doi:10.1016/i.nahs.2006.10.012

[10] A. M. Blokhin and D. L. Tkachev, "Stability of a Supersonic Flow about a Wedge with Weak Shock Wave," Sbornik: Mathematics, Vol. 200, No. 2, 2009, pp. 157-184.

[11] A. M. Blokhin, D. L. Tkachev and Y. Y. Pashinin, "The Strong Shock Wave in the Problem on Flow around Infinite Plane Wedge," Proceedings of the 11th international conference on hyperbolic problems, Springer-Verlag, Berlin, 2008, pp. 1037-1044.

[12] D. L. Tkachev and A. M. Blokhin, "Courant - Friedrich's Hypothesis and Stability of the Weak Shock," Proceedings of the 12th international conference on hyperbolic problems, Vol. 67, No. 2, 2009, pp. 958-966.

[13] R. Sakamoto, "Hyperbolic Boundary Value Problems," Iwanami Shoten, Tokyo, 1978. PMCid:1537516

[14] A. M. Blokhin, "Energy Integrals and their Applications to Problems of Gas Dynamics," Nauka, Novosibirsk, 1986.

[15] A. M. Blokhin, R. S. Bushmanov and D. L. Tkachev, "The Lopatinski Condition in the Problem of Normal Gas Flow around the Wedge," Preprint 271, Sobolev Institute of Mathematics, Novosibirsk, 2011. PMCid:3308588 